\title{
The high velocity outflow in NGC 6334 I
}

\author{
S. Leurini, P. Schilke, B. Parise, F. Wyrowski, R. Güsten, and S. Philipp
}

Max-Planck-Institut für Radioastronomie, Auf Dem Hügel 69, 53121 Bonn, Germany
e-mail: [sleurini; schilke; bparise; wyrowski ; rguesten; sphilipp]@mpifr-bonn.mpg.de

Received 31 March 2006 / Accepted 27 April 2006

\begin{abstract}
Aims. We observed the high velocity outflow originating from NGC 6334 I in several CO transitions with the APEX telescope, with the goal of deriving the physical parameters of the gas.

Methods. Using an LVG analysis, we studied line ratios between the $\mathrm{CO}(3-2), \mathrm{CO}(4-3)$, and $\mathrm{CO}(7-6)$ data as a function of the density and of the kinetic temperature of the gas. An upper limit on the CO column density is derived by comparison with ${ }^{13} \mathrm{CO}$ data. Results. We constrained the temperature to be higher than $50 \mathrm{~K}$ and the $\mathrm{H}_{2}$ density to values higher than $n \sim 10^{4} \mathrm{~cm}^{-3}$ towards the peak position in the red lobe, while $T>15 \mathrm{~K}$ and $n>10^{3} \mathrm{~cm}^{-3}$ are derived towards the peak position in the blue lobe. The physical parameters of the outflow, its mass and its energetics, have been computed using the temperatures derived from this analysis.

Conclusions. We conclude that high kinetic temperatures are present in the outflow and traced by high excitation CO lines. Observations of high- $J$ CO lines are thus needed to infer reliable values of the kinetic temperatures and of the other physical parameters in outflows.
\end{abstract}

Key words. star formation - ISM: jets and outflows

\section{Introduction}

Despite molecular outflows being a well-studied phenomenon associated with young stellar objects of all masses, many questions on their driving mechanisms and on their interaction with the surrounding material are still unanswered. The case of massive outflows, which only recently have been investigated at high spatial resolution (e.g. Beuther et al. 2002a; Sollins et al. 2004), is even more problematic. Usually, low rotational (J) CO transitions are used to trace molecular outflows and derive their physical parameters and energetics. However, these lines trace only cold gas, while high- $J$ transitions (e.g. Beuther et al. 2002a) have also been detected, clearly pointing to hot, dense gas associated with the outflow. High resolution observations of high- $J$ $\mathrm{CO}$ transitions are needed to get a better understanding of the phenomenon.

The region NGC 6334, located at a distance of $1.7 \mathrm{kpc}$ from the Sun (Neckel 1978), harbours a series of luminous starforming regions at various stages of evolution. NGC 6334 I is the dominant source at (sub)millimeter wavelengths (Sandell 2000), with a compact HII region at its center (Carral et al. 1997). Sub-mm line surveys (Sandell 1994; McCutcheon et al. 2000; Thorwirth et al. 2003; Schilke et al., this volume) reveal rich molecular line emission from a dense, hot core of size $\leq 10^{\prime \prime}$. Two outflows originate from it (Bachiller \& Cernicharo 1990; McCutcheon et al. 2000).

In this Letter, we present high frequency ${ }^{13} \mathrm{CO}$ and $\mathrm{CO}$ observations of the main outflow originating from NGC $6334 \mathrm{I}$. The physical parameters of the outflow are derived by a Large Velocity Gradient (LVG) analysis of several CO transitions.

\section{Observations}

The observations of NGC $6334 \mathrm{I}\left(\alpha_{\mathrm{J} 2000}=17: 20: 53.35, \delta_{\mathrm{J} 2000}=\right.$ -35:47:01.50) were performed with the Atacama Pathfinder Experiment $12 \mathrm{~m}$ telescope (APEX ${ }^{1}$, Güsten et al. 2006, this volume) during June, September, and November, 2005. The APEX-2A (Risacher et al. 2006, this volume) and FLASH (Heyminck et al. 2006, this volume) receivers were used to map the source in $\mathrm{CO}(3-2)$ and $\mathrm{CO}(4-3)$. The second channel of the FLASH receiver was tuned to $\mathrm{CO}(7-6)$; we performed long integration observations of the two positions where the blue and redshifted emission in $\mathrm{CO}(4-3)$ is strongest, at the offset positions $\left(-10^{\prime \prime},-14^{\prime \prime}\right)$ and $\left(17.5^{\prime \prime}, 14^{\prime \prime}\right)$. Additional observations of the same two positions in ${ }^{13} \mathrm{CO}(3-2)$ were performed in November 2005 with the APEX-2A receiver. All observations were taken in the lower side band. For the $\mathrm{CO}(4-3)$ and $\mathrm{CO}(7-6)$ observations, the system temperatures are around $800 \mathrm{~K}$ and $3400 \mathrm{~K}$, at $461 \mathrm{GHz}$ and $807 \mathrm{GHz}$ respectively, while the system temperatures of the APEX-2A receiver are of the order of $260 \mathrm{~K}$ for $\mathrm{CO}(3-2)$ and $480 \mathrm{~K}$ for ${ }^{13} \mathrm{CO}(3-2)$. The system temperatures of the ${ }^{13} \mathrm{CO}(3-2)$ are higher, as they were performed in November 2005 when the source was already in the Sun avoidance limit and was observed at low declination $\left(<20^{\circ}\right)$ after sunset. Pointing was checked on the continuum of NGC 6334 I itself, during the $\mathrm{CO}(4-3)$ observations, and was found to be accurate to $\sim 5^{\prime \prime}$. On the other hand, problems with the pointing were encountered during the $\mathrm{CO}(3-2)$ run and the data show a systematic shift of $\sim 8^{\prime \prime}$ with respect to the

1 This publication is based on data acquired with the Atacama Pathfinder Experiment (APEX). APEX is a collaboration between the Max-Planck-Institut für Radioastronomie, the European Southern Observatory, and the Onsala Space Observatory. 


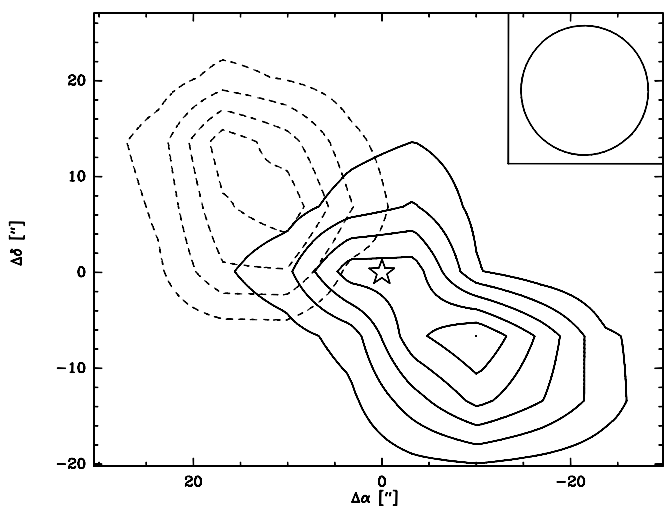

Fig. 1. Map of the $\mathrm{CO}(4-3)$ emission in the $7<v<55 \mathrm{~km} \mathrm{~s}^{-1}$ (red, dashed line) and $-78<v<-15 \mathrm{~km} \mathrm{~s}^{-1}$ (blue, solid line) ranges. Contours are from 100 to $600 \mathrm{~K} \mathrm{~km} \mathrm{~s}^{-1}$ by steps of 100 . The star marks the NGC 6334 I position; the beam $\left(\sim 13.5^{\prime \prime}\right)$ is indicated in the top right corner.

$\mathrm{CO}(4-3)$ observations. Alignment of the $\mathrm{CO}(3-2)$ map to the $\mathrm{CO}(4-3)$ is possible since several molecules are detected in the two bands that have high excitation conditions and peak on the hot core. We estimate the accuracy to be good to 2-3", since it was based on the alignment of hot core lines, which trace the (within our beam) point like hot core.

The FFTS spectrometer (Klein et al. 2006, this volume) was used with resolutions of $0.1 \mathrm{~km} \mathrm{~s}^{-1}$ for $\mathrm{CO}(3-2), 0.3 \mathrm{~km} \mathrm{~s}^{-1}$ for $\mathrm{CO}(4-3)$ and $\mathrm{CO}(7-6)$, and $0.4 \mathrm{~km} \mathrm{~s}^{-1}$ for ${ }^{13} \mathrm{CO}(3-2)$. The calibration was performed by using the APECS software (Muders et al. 2006, this volume). Antenna temperatures were converted to main-beam temperatures by using forward and beam efficiencies of 0.97 and 0.74 at $345 \mathrm{GHz}, 0.95$ and 0.60 at $464 \mathrm{GHz}$, and 0.95 and 0.43 at $809 \mathrm{GHz}$.

The $\mathrm{CO}(4-3)$ map is shown in Fig. 1, while spectra towards the $\left(-10^{\prime \prime},-14^{\prime \prime}\right)$ and $\left(17.5^{\prime \prime}, 14^{\prime \prime}\right)$ positions are presented in Figs. 2 and 3. Bachiller \& Cernicharo (1990) detected emission at $-100 \mathrm{~km} \mathrm{~s}^{-1}$ in the $\mathrm{CO}(2-1)$ spectra; they did not identify it with a $\mathrm{CO}$ velocity component, even though they could not assign the feature to any other molecule, because it peaks at the hot core position. The detection of a similar feature at $-100 \mathrm{~km} \mathrm{~s}^{-1}$ in our $\mathrm{CO}(3-2)$ spectra (Fig. 3) supports the interpretation that this is indeed another $\mathrm{CO}$ high velocity component. Alternatively, the feature can be identified as $\mathrm{CH}_{3} \mathrm{OH}$ $\left(16_{1} \rightarrow 15_{2}, E_{\text {low }} \sim 316 \mathrm{~K}\right)$ as it peaks on the hot core. A possible interpretation is that this feature is a CO component, since high excitation methanol lines are unlikely to be detected at positions distant from hot cores, and that contamination with $\mathrm{CH}_{3} \mathrm{OH}$ happens at the hot core position.

McCutcheon et al. (2000) show that the absorption at $6.5 \mathrm{~km} \mathrm{~s}^{-1}$ (see Figs. 2 and 3) is real, not coming from the off position used for position switching.

\section{Physical parameters of the outflow}

To determine the physical parameters in the outflow, we used the CO observations and a spherically symmetric LVG statistical equilibrium code, with the cosmic background as the only radiation field. To compare the observations at the three different frequencies with the LVG results, we smoothed the $\mathrm{CO}(4-3)$ and $\mathrm{CO}(7-6)$ data to the same spatial resolution of the $\mathrm{CO}(3-2)$, which is $18^{\prime \prime}$. To reduce the noise level in the spectra, we resampled them to a resolution of $5 \mathrm{~km} \mathrm{~s}^{-1}$.

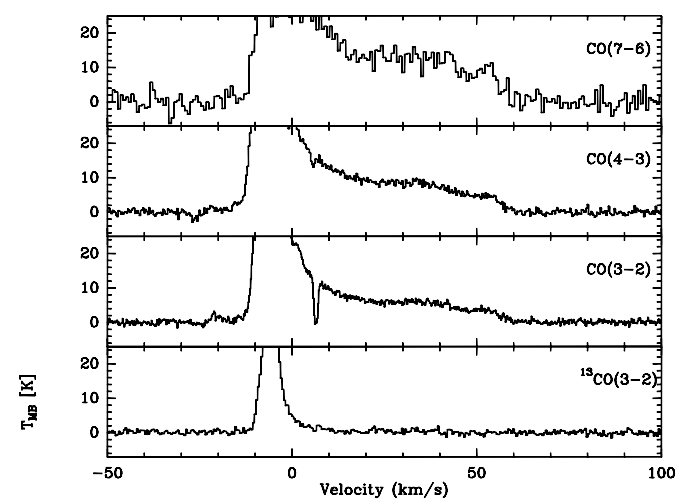

Fig. 2. $\mathrm{CO}(J=3,4,7)$ and ${ }^{13} \mathrm{CO}(3-2)$ spectra at the offset (17.5", $\left.14^{\prime \prime}\right)$ from the reference position $\left(\alpha_{\mathrm{J} 2000}=17: 20: 53.35, \delta_{\mathrm{J} 2000}=\right.$ $-35: 47: 01.50)$. The $\mathrm{CO}(7-6)$ and $\mathrm{CO}(4-3)$ spectra are smoothed to the $\mathrm{CO}(3-2)$ spatial resolution.

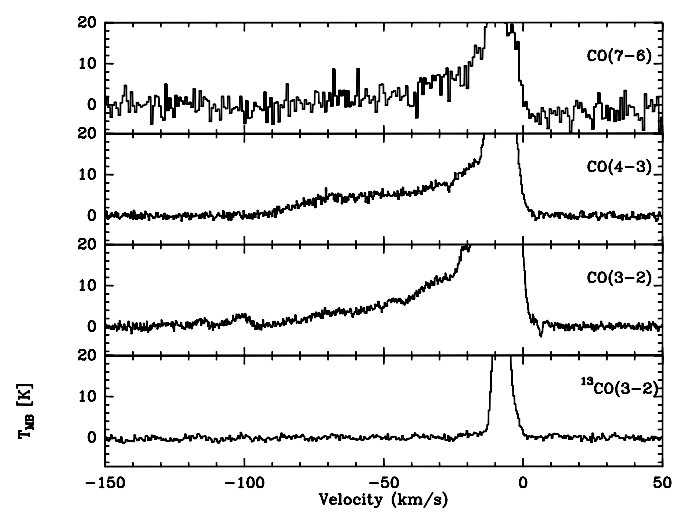

Fig. 3. $\mathrm{CO}(J=3,4,7)$ and ${ }^{13} \mathrm{CO}(3-2)$ spectra at the offset $\left(-10^{\prime \prime}\right.$, $\left.-14^{\prime \prime}\right)$ from the reference position $\left(\alpha_{\mathrm{J} 2000}=17: 20: 53.35, \delta_{\mathrm{J} 2000}=\right.$ $-35: 47: 01.50)$. The $\mathrm{CO}(7-6)$ and $\mathrm{CO}(4-3)$ spectra are smoothed to the $\mathrm{CO}(3-2)$ spatial resolution.

The non-detection of the ${ }^{13} \mathrm{CO}(3-2)$ transition in the wings gives an upper limit to the $\mathrm{CO}$ column density of $10^{17} \mathrm{~cm}^{-2}$ for the $\left(-10^{\prime \prime},-14^{\prime \prime}\right)$ and $\left(17.5^{\prime \prime}, 14^{\prime \prime}\right)$ positions. In this calculation, we used the relation between ${ }^{12} \mathrm{C}$ and ${ }^{13} \mathrm{C}$ given by Wilson \& Rood (1994) and a galactocentric distance of $6.8 \mathrm{kpc}$ (Kraemer et al. 1998). All models were run for this value of the column densiy. At this column density, the CO transitions are optically thin, with the exception of $\mathrm{CO}(3-2)$, which is optically thick for $T<50 \mathrm{~K}, n\left(\mathrm{H}_{2}\right)<10^{4} \mathrm{~cm}^{-3}$. Hence, our results should not depend much on the column density used in the calculations. The code inputs are the density and the kinetic temperature of the gas. Since a direct estimate of the latter is not available, we ran models for several densities and temperatures.

Source sizes play an important role in comparing the observations to the results of the LVG simulations, as the main beam temperature of a transition is equal to $\frac{s^{2}}{s^{2}+\Omega^{2}} \times T_{1}$, where $s$ and $\Omega$ are the source size and the beam size in $\operatorname{arcsec}$, and $T_{1}$ is the line intensity from the LVG analysis. In our case, the beam is the same for all the transitions, but the source sizes may differ as the three lines have different excitation conditions. Therefore, line ratios may also be affected by the uncertainties in the beam filling factors. In the following, we will analyse the line ratios between the three transitions (upper $J$ divided by lower $J$ ), assuming that all transitions are emitted by the same volume of gas. These are lower limits to the effective line ratios, as less excited transitions are likely to come from more extended regions. 


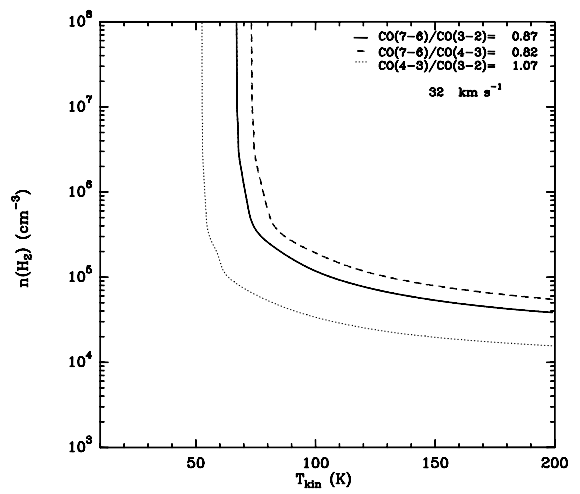

Fig. 4. Line ratios as a function of temperature and density toward the $\left(17.5^{\prime \prime}, 14^{\prime \prime}\right)$ position.

In Fig. 4, the three different line ratios at $\left(17.5^{\prime \prime}, 14^{\prime \prime}\right)$, $v_{\text {lsr }}=32 \mathrm{~km} \mathrm{~s}^{-1}$, are plotted as a function of density and kinetic temperature. Since we have ignored the effects of beam dilution, which are likely to be important at least for the $\mathrm{CO}(7-6) / \mathrm{CO}(3-2)$ line ratio, we can only estimate lower limits to the gas parameters. We find that the temperature must be higher than $50 \mathrm{~K}$, while the density is of the order of $10^{4} \mathrm{~cm}^{-3}$, equivalently a thermal pressure of $5 \times 10^{5} \mathrm{~cm}^{-3} \mathrm{~K}$. Moreover, as the line ratios between the $\mathrm{CO}(7-6)$ line and the other transitions show, there is also a denser, warmer component, from which the $\mathrm{CO}(7-6)$ line is emitted. As already discussed, no corrections for the different beam filling factors are applied, and the values we used for the line ratios are likely to be lower limits. Therefore, the values of $n=7 \times 10^{4} \mathrm{~cm}^{-3}$ and $T=70 \mathrm{~K}$ (corresponding to $P_{\text {thermal }} \sim 5 \times 10^{6} \mathrm{~cm}^{-3} \mathrm{~K}$ ) that we derived for the component traced by $\mathrm{CO}(7-6)$ are lower limits (consistent with other studies of high- $J$ transitions in outflows, e.g. Beuther et al. 2002a). Taking into account a calibration error of 0.2 for each ratio, we find $30<T<55 \mathrm{~K}, 7 \times 10^{3}<n<2 \times 10^{4} \mathrm{~cm}^{-3}$. For the warmer component traced by $\mathrm{CO}(7-6) / \mathrm{CO}(4-3) 55<T<95 \mathrm{~K}$, $3 \times 10^{4}<n<1 \times 10^{5} \mathrm{~cm}^{-3}$. The results for the other velocity channels are very similar, as the line ratios between the different $\mathrm{CO}$ transitions do not significantly change at this position (Fig. 2).

For the analysis on the $\left(-10^{\prime \prime},-14^{\prime \prime}\right)$ position, we only used the $\mathrm{CO}(4-3) / \mathrm{CO}(3-2)$ ratio, since the signal-to-noise ratio in the $\mathrm{CO}(7-6)$ spectrum is not very high in the wings $(\sim 3)$. The lower limits on the temperature and the density of the gas vary along the wings, ranging between 15 and $50 \mathrm{~K}, 2 \times 10^{3}$ and $2 \times 10^{4} \mathrm{~cm}^{-3}$ (see Figs. 5a and b). Taking into account the uncertainties on the calibration, the lower limits we derive range between 10 and $30 \mathrm{~K}$ and $n<10^{4} \mathrm{~cm}^{-3}$ for $v_{\mathrm{lsr}}=-48 \mathrm{~km} \mathrm{~s}^{-1}$, and $20<T<56 \mathrm{~K}$ and $4 \times 10^{3}<n<3 \times 10^{4} \mathrm{~cm}^{-3}$ for $v_{\text {lsr }}=-58 \mathrm{~km} \mathrm{~s}^{-1}$.

The difference in temperatures and densities derived for the red and blue lobes may be caused by the (pre-existing) density structure of the molecular cloud into which the outflow impinges and with which it interacts. Continuum maps of the region (Sandell 2000) support this scenario, since the continuum emission is extended to the north of NGC $6334 \mathrm{I}$.

The CO column density of the high velocity gas can be derived from the integrated intensity, by assuming that the level populations are in LTE at the kinetic temperature derived in our calculations ( $30 \mathrm{~K}$ for the blue lobe, $50 \mathrm{~K}$ for the red one). To avoid contamination from the core emission, we integrate between 10 and $59 \mathrm{~km} \mathrm{~s}^{-1}$ for the redshifted gas, and -88 to $-22 \mathrm{~km} \mathrm{~s}^{-1}$ for the blueshifted gas, which results in lower

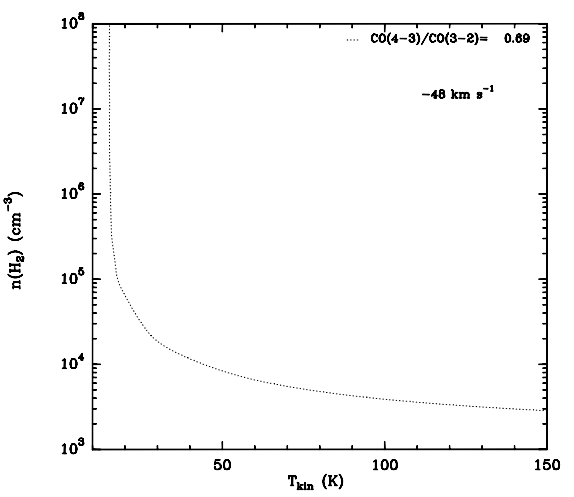

(a)

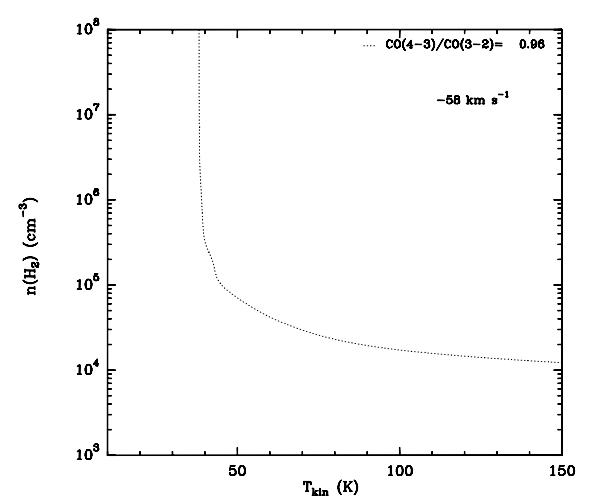

(b)

Fig. 5. $\mathrm{CO}(4-3) / \mathrm{CO}(3-2)$ line ratio as a function of temperature and density for two velocity channels toward the $\left(-10^{\prime \prime},-14^{\prime \prime}\right)$ position.

limits to the derived masses. The momentum $p$, the energy $E$, and the characteristic time scale $t$ can be derived assuming that the outflow is a constant velocity flow with true velocity equal to the maximum velocity observed (e.g. Lada 1985; Beuther et al. 2002 b). Finally, the mass entrainment rate $\dot{M}_{\text {tot }}$, the mechanical force $F$, and the mechanical luminosity $L$ can be derived from the other parameters. Assuming that the inclination angle between the outflow and the line of sight is $\theta$, the following relations are used (from Beuther et al. 2002b, but corrected for the inclination angle $\theta$ ):

$N\left(\mathrm{H}_{2}\right)=10^{4} \frac{6.97 \times 10^{15}}{v^{2} \mu^{2}} T_{\mathrm{ex}} \mathrm{e}^{\left(E_{\mathrm{u}} / T_{\mathrm{ex}}\right)} \int T_{\mathrm{CO}(4-3)} \mathrm{d} v$

$M_{\mathrm{tot}}=\frac{1}{\cos \theta}\left(N_{\mathrm{b}} \times \operatorname{area}_{\mathrm{b}}+N_{\mathrm{r}} \times \operatorname{area}_{\mathrm{r}}\right) m_{\mathrm{H}_{2}}$

$p=\frac{1}{\cos \theta}\left(M_{\mathrm{b}} \times v_{\text {max }_{\mathrm{b}}}+M_{r} \times v_{\text {max }_{\mathrm{r}}}\right)$

$E=\frac{1}{\cos ^{2} \theta}\left(\frac{1}{2} M_{\mathrm{b}} \times v_{\max _{\mathrm{b}}}^{2}+\frac{1}{2} M_{\mathrm{r}} \times v_{\max _{\mathrm{r}}}^{2}\right)$

$t=\frac{r}{2 \sin \theta} \times \frac{\cos \theta}{v_{\text {max }_{\mathrm{b}}}+v_{\text {max }_{\mathrm{r}}}}$

$F=\frac{p}{t}$

$\dot{M}_{\mathrm{tot}}=\frac{M_{\mathrm{tot}}}{t}$

$L=\frac{E}{t}$. 
Table 1. Physical parameters of the outflow.

\begin{tabular}{lccc}
\hline \hline$N_{\mathrm{b}}$ & \multicolumn{3}{c}{$1.9 \times 10^{21} \mathrm{~cm}^{-2}$} \\
$N_{\mathrm{r}}$ & no corr. & $\theta=40^{21} \mathrm{~cm}^{-2}$ & $\theta=70^{\circ}$ \\
$M_{\mathrm{b}}\left[M_{\odot}\right]$ & 1.1 & 1.5 & 3.2 \\
$M_{\mathrm{r}}\left[M_{\odot}\right]$ & 0.8 & 1.2 & 2.4 \\
$M_{\mathrm{tot}}\left[M_{\odot}\right]^{a}$ & 1.9 & 2.7 & 5.6 \\
$t\left[10^{3} \mathrm{yr}\right]$ & 4.4 & 4.5 & 1.6 \\
$\left.p\left[M_{\odot} \mathrm{km} \mathrm{s}\right]^{-1}\right]^{b}$ & 140 & 200 & 410 \\
$E\left[10^{47} \mathrm{erg}\right]$ & 1.0 & 2.1 & 8.9 \\
$F_{\mathrm{m}}\left[M_{\odot} \mathrm{km} \mathrm{s}^{-1} \mathrm{yr}^{-1}\right]$ & 0.03 & 0.04 & 0.3 \\
$L_{\mathrm{m}}\left[L_{\odot}\right]$ & 780 & 1550 & 1800 \\
$\dot{M}_{\mathrm{tot}}\left[10^{-3} M_{\odot} \mathrm{yr}^{-1}\right]$ & 1.9 & 2.6 & 15 \\
\hline
\end{tabular}

${ }^{a}$ Bachiller \& Cernicharo (1990): $2.3 M_{\odot}$; McCutcheon et al. (2000): $4.8 M_{\odot}$

${ }^{b}$ Bachiller \& Cernicharo (1990): $92 M_{\odot} \mathrm{km} \mathrm{s}^{-1}$; McCutcheon et al. (2000): $360 M_{\odot} \mathrm{km} \mathrm{s}^{-1}$.

Estimates of the physical parameters in the outflow from NGC 6334 I have already been derived from the analysis of $\mathrm{CO}(2-1)$ and $\mathrm{CO}(3-2)$ observations. Both Bachiller \& Cernicharo (1990) and McCutcheon et al. (2000) do not apply any corrections for the inclination angle to their values. In Table 1, the parameters are given for no corrections for the inclination angle and for $\theta=45^{\circ}$ and $70^{\circ}$, as the small overlap between the redshifted and the blue emission and the emission at high velocity indicate that $\theta$ is closer to $90^{\circ}$ than to $0^{\circ}$. For comparison, results from Bachiller \& Cernicharo (1990) and McCutcheon et al. (2000) are also given. Our values are in good agreement with previous studies; however, the mass we derive, and therefore other parameters also, are smaller than what both Bachiller \& Cernicharo (1990) and McCutcheon et al. (2000) had derived, due to the higher kinetic temperature we use for the red lobe. Morever, high- $J$ transitions trace only the warm gas, while low- $J$ lines also trace a colder component. Both this study and McCutcheon et al. (2000) make use of the maximum observed velocity in the outflow to derive the energetics of the outflow, while Bachiller \& Cernicharo (1990) use the mean velocity. The estimate of the mass is also affected by other uncertainties; the observed area of the outflow depends on the transitions used in the calculations and the excitation temperature used in Eq. (1) has been derived for one position per lobe. Gibb et al. (2003) also found lower outflow masses in $\mathrm{CO}(3-2)$ than in $\mathrm{CO}(1-0)$; Cabrit \& Bertout (1990) have shown that masses derived using Eq. (2) are upper limits to their true value.

\section{Conclusions}

We report observations in high excitation CO lines towards the high velocity outflow from NGC 6334 I. By using an LVG analysis, we have constrained the temperatures to be higher than $50 \mathrm{~K}$ and the $\mathrm{H}_{2}$ density to values higher than $n \sim 10^{4} \mathrm{~cm}^{-3}$ towards the peak position in the red lobe, $T>15 \mathrm{~K}$ and $n>10^{3} \mathrm{~cm}^{-3}$ towards the peak position in the blue lobe. The physical parameters of the outflow, its mass and its energetics, have been computed using the temperatures derived from this analysis. Often (e.g. Beuther et al. 2002b; Kim \& Kurtz 2006), low temperatures are assumed in computing outflow masses; our analysis has shown that high kinetic temperatures are present, and traced by high excitation $\mathrm{CO}$ lines, while they are not found when only low energy transitions are available. High spatial resolution maps of $\mathrm{CO}$ transitions at high- $J$ are needed to infer reliable values of the kinetic temperatures and of the other physical parameters in the outflow.

Acknowledgements. We would like to thank the anonymous referee for the pront reply. BP is grateful to the Alexander von Humboldt Foundation for a Research Fellowship.

\section{References}

Bachiller, R., \& Cernicharo, J. 1990, A\&A, 239, 276

Beuther, H., Schilke, P., Gueth, F., et al. 2002a, A\&A, 387, 931

Beuther, H., Schilke, P., Sridharan, T. K., et al. 2002b, A\&A, 383, 892

Cabrit, S., \& Bertout, C. 1990, ApJ, 348, 530

Carral, P., Kurtz, S. E., Rodriguez, L. F., de Pree, C., \& Hofner, P. 1997, ApJ, 486, L103

Gibb, A. G., Hoare, M. G., Little, L. T., \& Wright, M. C. H. 2003, MNRAS, 339, 1011

Kim, K.-T., \& Kurtz, S. E. 2006, ApJ, accepted [astro-ph/0601532]

Kraemer, K. E., Jackson, J. M., \& Lane, A. P. 1998, ApJ, 503, 785

Lada, C. J. 1985, ARA\&A, 23, 267

McCutcheon, W. H., Sandell, G., Matthews, H. E., et al. 2000, MNRAS, 316, 152

Neckel, T. 1978, A\&A, 69, 51

Sandell, G. 1994, MNRAS, 271, 75

Sandell, G. 2000, A\&A, 358, 242

Sollins, P. K., Hunter, T. R., Battat, J., et al. 2004, ApJ, 616, L35

Thorwirth, S., Winnewisser, G., Megeath, S. T., \& Tieftrunk, A. R. 2003, in Galactic Star Formation Across the Stellar Mass Spectrum, ASP Conf. Ser., 287, 257

Wilson, T. L., \& Rood, R. 1994, ARA\&A, 32, 191 